\title{
LA GESTIÓN PARA LA MEJORA DE PROCESOS: PROPUESTA PARA EL CAMBIO Y EVALUACIÓN DE LOS PROCESOS LOGÍSTICOS EN EL SECTOR DE LAS TELECOMUNICACIONES*
}

\author{
Management for the improvement of business processes: \\ Proposal for the change and evaluation of logistics processes \\ in the telecommunications sector
}

\author{
ANGÉLICA LIZETH MARTÍNEZ RAMÍREZ", CRISTIAN CAMILO URREGO BARRIOS², \\ EVER ÁNGEL FUENTES ROJAS ${ }^{3}$
}

Recibido:15 de diciembre de 2020. Aceptado:15 de enero de 2021

DOI: http://dx.doi.org/10.21017/rimci.2021.v8.n15.a90

\begin{abstract}
Resumen
Para responder a los cambios del entorno, la gestión por procesos aporta una visión y unas herramientas con las que se puede cambiar las metas de la empresa y mejorar los métodos de funcionamiento, es decir, los procesos [1], para hacer el trabajo más eficiente y adaptado a las necesidades de todos los usuarios. Los planteamientos de la gestión por procesos constituyen una propuesta ventajosa para el perfeccionamiento de la gestión, razón por la cual en el presente artículo, se propone un trabajo de estandarización de los procesos logísticos en una compañía del sector de las telecomunicaciones como solución para el cambio y aumento de la productividad. Para tal fin se plasma una metodología que se basa en un diagnóstico como consecuencia de una contextualización previa || y basado en aplicación de herramientas de análisis, una propuesta de mejora que involucra la estructuración, actualización, documentación de subprocesos y estudio de tiempos de trabajo, y finalmente un análisis de impacto de la investigación obteniendo como resultado una disminución del $11 \%$ de la documentación con ampliación del alcance de cada uno de los subprocesos y un $21 \%$ en los tiempos de ejecución de las actividades.
\end{abstract}

Palabras clave. Estandarización; gestión; estructuración; procesos; valor agregado

\begin{abstract}
Process management provides a vision and tools with which you can improve and redesign the workflow to make it more efficient and adapted to the needs of all users. Process management approaches are an advantageous proposal for the improvement of management, which is why in this article a work is proposed to standardize logistics processes in a company in the telecommunications sector as a solution for change. To this end, the methodology is based on a diagnosis as a result of a pre-contextualization and based on the application of analysis tools, a proposal for improvement involving structuring, updating, thread documentation and study of working times, and finally an analysis of research impact resulting in an $11 \%$ decrease in documentation and expanding the scope of each of the threads and $21 \%$ in the execution times of the activities.
\end{abstract}

Key words. Standardization; management; structuring; processes and value added.

* Artículo desarrollado con base en el proyecto de Estandarización de los procesos logísticos en una compañía colombiana del sector de las telecomunicaciones, llevado a cabo desde el mes de enero de 2019 hasta el mes de marzo de 2021, por estudiantes del programa de Ingeniería Industrial de la Universidad Libre, sede Bogotá-Colombia

1 Ingeniería Industrial, Universidad Libre Bogotá - Colombia. Correo electrónico: angelical-martinezr@unilibre.edu.co ORCID: https:// orcid.org/0000-0001-5100-1757

2 Ingeniería Industrial, Universidad Libre Bogotá - Colombia. Correo electrónico: cristianc-urregob@unilibre.edu.co.

3 MBA., profesor de la Facultad de Ingeniería, Universidad Libre Bogotá - Colombia. Correo electrónico: ever.fuentes@unilibre.edu.co ORCID: https://orcid.org/0000-0001-9671-5884 


\section{INTRODUCCIÓN}

E SECTOR de las telecomunicaciones ha venido acelerándose con el pasar del tiempo y al ritmo de las exigencias de evolución de la tecnología que asecha al mundo entero. Colombia, si bien, es un país con más de 23 millones de habitantes sin beneficios de conectividad, más de 21 millones cuentan con servicios de banda ancha y telefonía, por lo que se ha registrado un crecimiento cerca al $30 \%$ de uso de los servicios de las telecomunicaciones mejorando notablemente indicadores y su aporte al PIB del país (representado en un $1.19 \%$ ) [2].

De acuerdo con un análisis del sector TIC realizado por el DANE (2019), la cadena de valor TIC en Colombia se compone de cinco grandes dimensiones [3]:

1. La infraestructura que soporta la utilización de los servicios y productos.

2. La fabricación y/o venta de los bienes TIC.

3. La producción de los servicios de telecomunicaciones, donde el servicio de Internet comienza a generar un nuevo negocio.

4. La industria de las plataformas digitales.

5. Un último componente transversal compuesto por el conjunto de Investigación, desarrollo e innovación necesarias para la continua evolución del sector.

Actualmente, se precisa que uno de los elementos anteriores resulta del uso y apropiación de las tecnologías (como las soluciones a necesidades que ha demostrado las cifras de la economía), principalmente por la masificación de servicios de banda ancha. Este componente hace referencia a la infraestructura (despliegue, instalación y administración) que soporta la utilización de servicios y productos TIC y que reflejan el alto crecimiento por el que han venido atravesando. Para el primer trimestre de 2015, se logró más diez (10) millones de conexiones de Banda Ancha, un 13,4\% más que en un periodo del año 2014, registrando 5.311.059 suscriptores de internet fijo y 5.413 .313 suscriptores de internet móvil, de los cuales el 38,7\%, lo hicieron a través del operador Claro [4].
Debido al avance del sector y la expansión dada en coberturas, en el mercado han penetrado grandes empresas de servicios de comunicaciones como lo son Claro con una participación del $33.7 \%$ para el año 2019, Tigo-Une (20.2\%) y Movistar (15.3\%), que ahora han entrado a competir con otras más pequeñas que lograron de algún modo posicionarse muy bien gracias a su asequibilidad y calidad de servicio. Con base en esto, el sector se ha visto en la necesidad de implementar otras instituciones que soporten la infraestructura para la instalación y distribución de servicios y además ofrecer otros de consultoría, implementación de proyectos y comercialización que ayuden a controlar y supervisar la amplia demanda que ellos generan.

Con la entidad de telecomunicaciones, ubicada en la ciudad de Bogotá, se entendió de manera más precisa el trabajo que requiere estar detrás de este sector y los procesos que se encuentran implicados para brindar un servicio eficiente tanto a clientes potenciales como a los usuarios finales. Con base en esto, se trabajó en la gestión por procesos directamente desde Logística, una de las áreas de apoyo en la cadena de valor de la compañía; con el fin de mejorar y actualizar todas las actividades para finalmente disponer de un servicio eficaz y de acuerdo a la demanda del mercado.

El trabajo de campo en la entidad consistió en aplicar un diagnóstico de entrada al estado de los procesos. Posteriormente, con los resultados obtenidos, estos se mejoraron y se actualizaron mediante la documentación necesaria exigida también por norma ISO. Se aplicó una metodología de toma de tiempos con el objetivo de determinar un estándar para cada una de las actividades del área.

Posteriormente, se establecieron indicadores de gestión y se aplicó un modelo para calcular los costos de calidad y no calidad. Con el desarrollo de este proyecto, se espera que la entidad del sector de las telecomunicaciones, disponga de unos procesos documentados y estandarizados para el área, para que así pueda identificar aquellas actividades que no generan valor agregado a su cadena de valor.

\section{Problema de investigación}

Esta empresa con más de 15 años de labor en el sector de las telecomunicaciones, cuenta con el apo- 
yo de personal experto en la industria y gracias a su trabajo y clientes potenciales como Claro, ha crecido exponencialmente y equivalente a ello sus procesos.

Cuentan con un macro-proceso: Gestión de Abastecimiento, donde se abarcan las áreas de compras, contratos, transporte, mantenimiento y finalmente, logística. Esta última, está directamente vinculada con los clientes (internos y externos) ya que a través de ella es posible tener conocimiento de los requisitos de la operación y satisfacer sus necesidades.

Esta área se encarga principalmente de:

- Abastecimiento, según requerimiento de proyectos y consumo de material/bienes

- Asignación y salidas de bienes de los almacenes

- Administración de inventario y conciliaciones de bienes

Cada dependencia de la compañía, es esencial para su funcionamiento y la interacción con sus clientes, ya que de ellos depende que cada proyecto se lleve a cabo de la forma más eficiente posible. Sin embargo, el área de Logística de esta empresa se encontraba en etapa de implementación en el mapa de procesos, por lo tanto, no estaba debidamente documentada y estandarizada, lo que pudo haber desencadenado en una etapa más larga de adaptación y aprendizaje por parte de los colaboradores, y por supuesto, al bajo índice de accionamiento de mejoras.

La compañía debe cumplir con normalizaciones que permitan certificar sus métodos de funcionamiento, sin embargo, no se encontraban estructurados de acuerdo a los requisitos (esencia de las actividades) y a las necesidades de los interesados. Esto podría representar un problema para la entidad ya que se dificulta lograr la productividad en un proceso que no es medible y que no tiene definido el deber ser del flujo de trabajo.

Finalmente, se planteó que para solucionar la problemática se deben establecer parámetros de estandarización para consolidar y ajustar los procesos logísticos de tal manera que se aumente la productividad.

\section{Estrategia METODOLÓGICA}

La determinación de la investigación para el proyecto es de tipo aplicada apoyada en fuentes documentales y con datos cualitativos debido a sus características, por lo que se toman decisiones basados en información descrita y experiencias de los interesados.

A partir de lo anterior, se planean cuatro fases para la ejecución del trabajo de campo.

Se procedió a iniciar con un análisis y contextualización de la compañía de telecomunicaciones con base en textos literarios y revisión de estados de arte con el objetivo de adquirir y alimentar el conocimiento de los autores en cuanto al caso de negocio implicado en la investigación. Esta etapa permitió tener un panorama del funcionamiento integral de la entidad, su normatividad y principalmente del flujo de trabajo relacionado con el área de logística. Como segunda etapa, se procedió a un trabajo de diagnóstico para conocer por medio de herramientas ingenieriles el estado actual del proceso y de esta manera identificar posibles oportunidades de mejoras, fallas y las causas relacionadas (Ver Fig. 1).

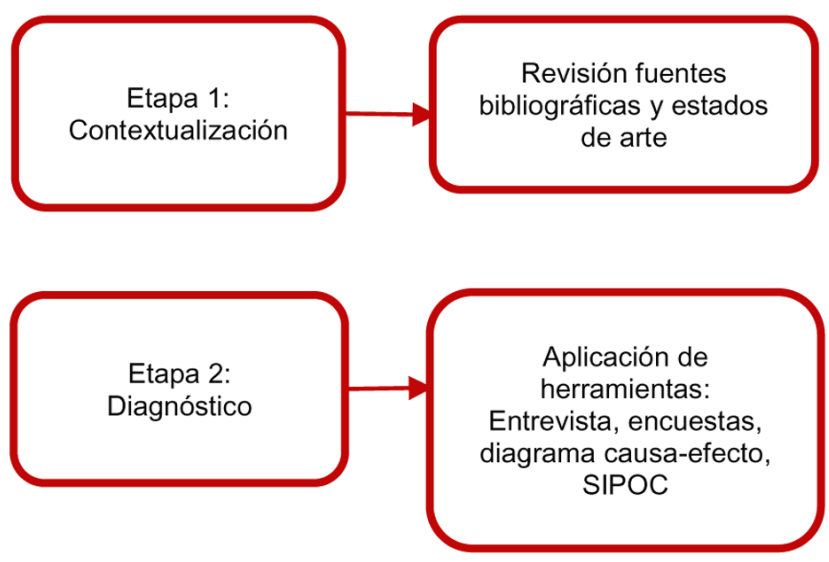

Fig. 1. Etapa 1 y 2 para la ejecución del proyecto. Fuente: Los autores, 2020

A consecuencia de las etapas previamente mencionadas y los resultados arrojados, se procedió a trabajar en el mejoramiento de las actividades logísticas de la compañía. Aquí, fue necesario realizar un análisis de la información suministrada por colaboradores, profesionales y dueños del proce- 
so, teniendo la oportunidad de conocer a fondo las necesidades de cada involucrado y tener al alcance los datos como insumo para generar valor en la propuesta objetivo.

Para conseguir un mayor impacto de la investigación en la empresa de telecomunicaciones, se realizó una medición del trabajo con el objetivo de aplicar una metodología de estudio de movimientos que ofreciera a la entidad los tiempos estándar y óptimos para la ejecución de cada una de las actividades logísticas. Se tomó como base la metodología utilizada en la guía para el estudio de cargas de trabajo de la Universidad Nacional (2013) [5], y a su vez se tuvo en cuenta las especificaciones definidas por la OIT para dar valoración del ritmo de trabajo teniendo presentes los factores que influyen en la eficiencia del desempeño del talento humano [6]. Así, se logra demostrar que se puede incrementar la productividad sin necesidad de generar inversiones en mano de obra.

Para ejecutar esta etapa, se trabajó junto con expertos profesionales y especializados en procesos logísticos, se aplicaron entrevistas para la toma de información y se definieron los tiempos estándar que podrían beneficiar la eficiencia de los procesos de la empresa (Ver Fig. 2).

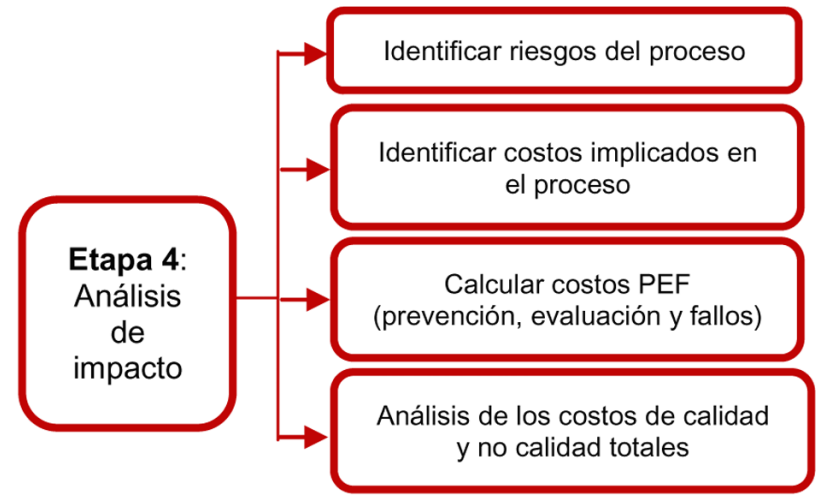

Fig. 2. Etapa 3 para la ejecución del proyecto. Fuente: Los autores, 2020.

Finalmente, y con el propósito de analizar el impacto de la investigación realizada, se consultó una metodología que se ajustara a los parámetros de la entidad e informara los costos de calidad y no calidad en caso de incurrir en riesgos del proceso.
La metodología utilizada para la evaluación de impacto se basó en el modelo PEF (Prevención, Evaluación y Fallos) definido por Dale y Plunkett (1995, citados en Gracia Villar, S., y Dzul López, L. 2007) [7], para determinar los costos de calidad y no calidad. Los de calidad son aquellos en los que se incurre para la mejora de los procesos de las empresas y garantizar la conformidad según norma y requisitos; los de no calidad son los asociados a fallos del sistema, producto y servicio [8].

Para la aplicación de dicha metodología dentro del estudio, se tomó como referencia, el trabajo realizado por Tapia, K. y Rodolfo, J. (2013) [9], el cuál consistió en la aplicación del modelo Prevención, Evaluación y Fallos, en una empresa del sector textil.

El modelo se basó en las siguientes fases [9]: (Ver Fig. 3).

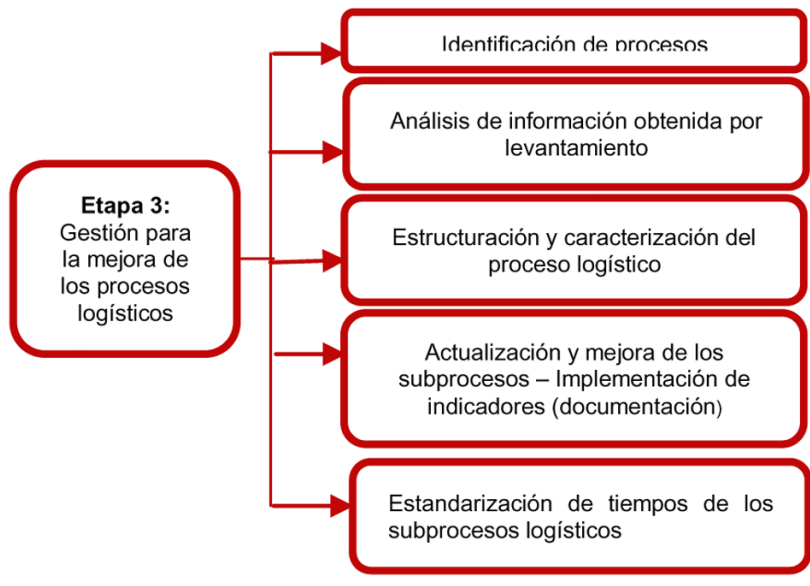

Fig. 3. Etapa 4 para la ejecución del proyecto. Fuente: Los autores, 2020

\section{A. Diagnóstico:}

Como herramientas para realizar el diagnóstico inicial del proceso de logística se emplearon entrevistas para conocer detalladamente la información que es tratada desde el área, documentos y aplicativos. Para complementar la información y obtener resultados más objetivos en la investigación se recurrió a realizar una encuesta a todos los colaboradores logísticos. Consolidando los resultados se obtuvo la siguiente información: 
- El diagrama de procesos del área de logística indicaba que se tenían 12 subprocesos de los cuales el $75 \%$ estaban documentados. (Se soporta esta información con los datos que se visualizan en el gráfico de abajo) (Ver Fig. 4).

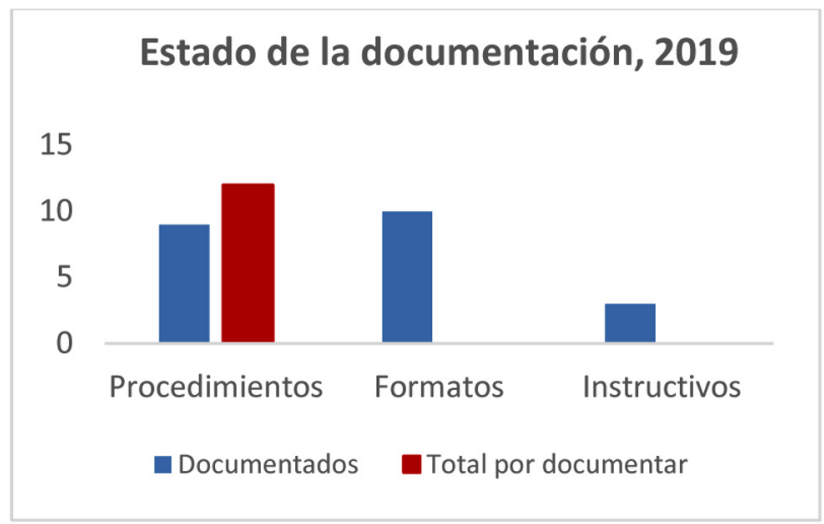

Fig. 4. Estado de documentación del área de logística para el año 2019. Fuente: Los autores, 2020

Según el flujo del proceso y varias reuniones con los interesados, la compañía tenía aprobados seis documentos que hacían referencia a las actividades que se llevan a cabo con los bienes suministrados por clientes. Sin embargo, a razón de la ejecución de sus proyectos y de todos los procesos de la compañía, la entidad administra bienes de terceros y bienes de su propiedad; por lo que se requería ampliar los objetivos y alcances para cada subproceso logístico [10].

- A la fecha, se tenían estructurados los siguientes indicadores para medir la eficiencia del proceso

- Gestión del almacenamiento

- Gestión de la recogida de los bienes del cliente

- Error en el pronóstico de consumo de los bienes de plan ferretero

- Exactitud de inventario

- Rotación de inventario

- El 37\% de los colaboradores desconocía la documentación o estructuración del proceso en el que estaban involucrados
- El 54\% del equipo logístico aprendió a ejecutar sus labores mediante la experticia adquirida o través de capacitaciones

- El 25\% de la muestra indica que los aplicativos utilizados no son eficientes

- La comunicación entre las áreas de la compañía según los encuestados es buena, lo que facilitaría la búsqueda de información interna brindando un apoyo en las diferentes actividades

- Las opciones de mejora detectadas por los encuestados durante el desempeño de su labor en la compañía van enfocadas a una mejor estructuración de los procesos teniendo en cuenta los aportes del personal para su optimización.

Con los resultados obtenidos del análisis de las entrevistas y encuestas, se construyó un diagrama Causa-Efecto con el que se buscó priorizar los problemas encontrados y así mismo, realizar un ejercicio de identificación de sus causas, obteniendo que existían fallos en el control interno causados por una situación de reestructuración general de la cadena de valor, así como fallos directos del área de logística por la falta de definición de la documentación necesaria para estructurar el proceso, la falta de proyección del desempeño de las actividades y su ejecución variable en los demás almacenes (a nivel nacional). El enfoque en los bienes del cliente, también fue motivo para dejar de lado la misión del proceso con otros bienes dentro de los cuales se administran los comprados por la compañía.

Por otra parte y para finalizar con la aplicación de herramientas que permitieran conocer las necesidades del proceso, se hizo uso del SIPOC que toma sus nombres de las siglas en inglés Supplier, Inputs, Process, Outputs y Customers, y es conocida como una técnica para identificar problemas de tal forma que se defina el alcance de la oportunidad de mejora y se asegure que esté alineado con los requerimientos de mayor nivel del cliente [11]. Con ayuda del SIPOC se identificaron los subprocesos y sus límites, se identificaron las salidas y sus clientes, las entradas y los proveedores y con ellos los requerimientos para cada uno. Gracias a este análisis se logró concluir que: 
- Era necesario reestructurar los subprocesos, y las actividades que se estaban ejecutando en el proceso de logística, además de definir aquellos relacionados con la administración de los bienes que son propiedad de la compañía

- Era necesario generar enfoque en la optimización del proceso y la estandarización para las demás regionales

- Era necesario establecer los indicadores dados para la medición y el control del proceso teniendo en cuenta los objetivos de calidad de la compañía

\section{B. Documentación:}

A través de la información recolectada mediante el diagnóstico, se logró definir para el área de logística los factores internos y externos, como también el flujo de actividades y los recursos necesarios en las entradas y salidas, identificando así los responsables de la ejecución de cada subproceso, apoyo y control. Todos estos elementos aportaron al desarrollo de la caracterización del proceso de Logística de la empresa de telecomunicaciones, basada en el "círculo de Deming", explicado por Gutiérrez [12] como un método de gran utilidad para estructurar y ejecutar proyectos de mejora de la calidad y la productividad en cualquier nivel jerárquico en una organización, tomando como base sus conceptos básicos: Planificar, plantear los objetivos, actividades y meta. Hacer, llevar a cabo un plan de trabajo. Verificar, comparar los resultados. Actuar, tomar acciones con respecto al rendimiento del proceso.

Partiendo de lo anterior y del producto de la retroalimentación realizada en conjunto con el área de logística, se establecen los objetivos, alcances, definiciones, responsabilidades, documentos de referencias, desarrollo de las actividades, diagramas de flujo, registros asociados, puntos de control y anexos para los subprocesos previamente determinados: Planeación de la demanda y solicitud de bienes, recepción y almacenamiento de bienes, entrega de bienes a los colaboradores, gestión de devoluciones, gestión de traslados, gestión de salidas, gestión de inventarios y conciliaciones de bienes.

En la revisión y actualización de la documentación implementada antes del proyecto se verificó su estado de vigencia y funcionalidad, de igual manera se validó aquellos registros que no contaban con una codificación y estaban fuera del listado maestro. Los resultados de la caracterización arrojaron datos porcentualmente positivos con respecto a los documentos del año 2019, por consiguiente, el cálculo del incremento se realizó por tipo documental: Procedimientos (disminuyó un $11 \%$ ), formatos (aumentó un 110\%) e instructivos (disminuyó en un 67\%). A continuación, se presenta el impacto después de la estandarización: (Ver Fig. 5).

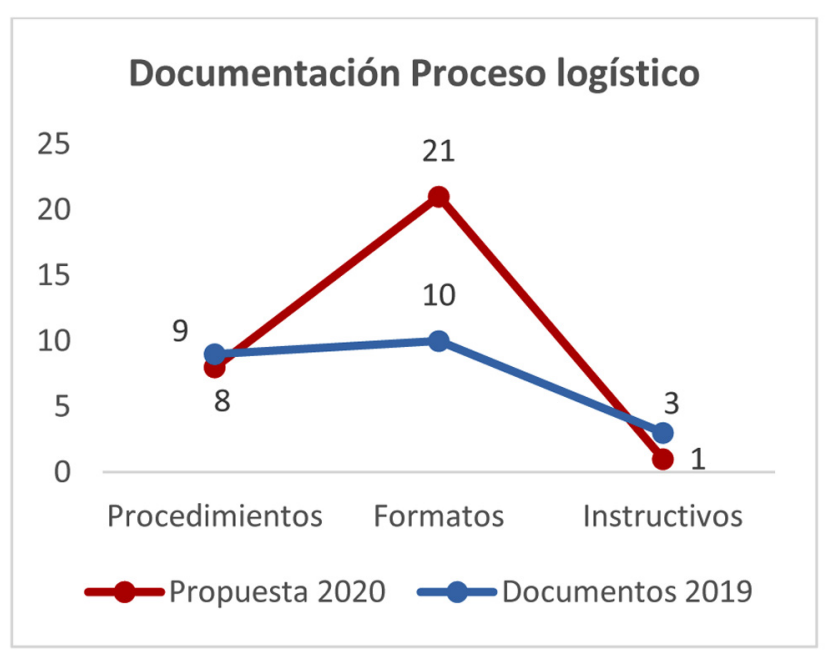

Fig. 5. Estado de documentación antes y después de la estandarización. Fuente: Los autores, 2020

Dentro de la caracterización se relacionan también los riesgos, controles, indicadores y aspectos ambientales que intervienen durante el avance del proceso. Según la afirmación de Pérez Jaramillo [13], un indicador de gestión es la expresión cuantitativa del comportamiento y desempeño de un proceso, cuya magnitud, al ser comparada con algún nivel de referencia, puede estar señalando una desviación sobre la cual se toman acciones, debe considerarse que las organizaciones no consiguen gestionar lo que no pueden medir, por esta razón se examinan los requisitos legales e institucionales para crear indicadores que generen valor y faciliten la identificación de oportunidades de mejora.

En la elaboración de indicadores se tuvo como foco los objetivos de calidad que representan la compañía y de acuerdo a esto se definieron los siguientes:

Rev. Ingeniería, Matemáticas y Ciencias de la Información Vol. 8 / Núm. 15 / enero - junio de 2021; pág. 33-44 
ANGÉLICA LIZETH MARTÍNEZ RAMÍREZ, CRISTIAN CAMILO URREGO BARRIOS, EVER ÁNGEL FUENTES ROJAS

- Calificación del cliente (indicador estratégico)

- Rotación de inventario (indicador de gestión)

- Exactitud del inventario físico (indicador de gestión)

- Exactitud del inventario fiscal (indicador operacional)

Además, se realizó una distribución de acuerdo a la gestión de los servicios logísticos referente a la eficiencia y eficacia. (Ver Fig. 6).

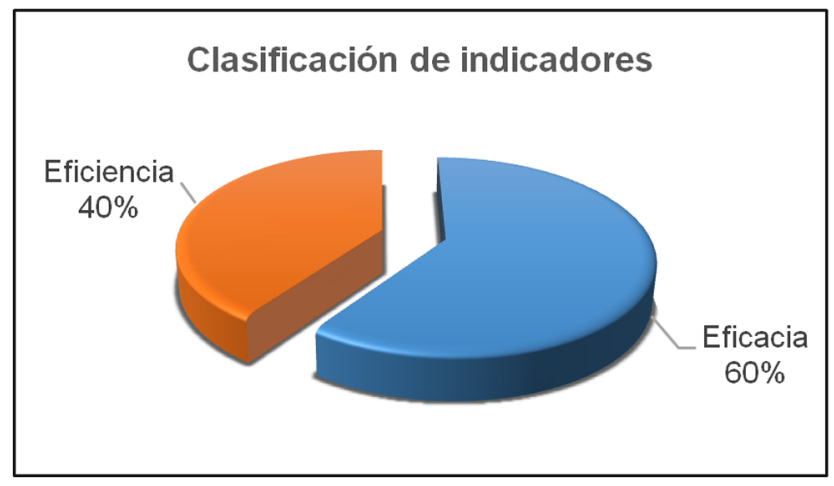

Fig. 6. Distribución por tipo de indicador. Fuente: Los autores, 2020

Es relevante comprender que la relación balanceada entre las evidencias documentales, sus controles y las mediciones de gestión por medio de indicadores proporcionan una comunicación transversal de las estrategias organizacionales, esto con el fin de ser direccionadas a una meta en común, por tal motivo se recomienda no solo comprenderlos como instrumentos de medición.

\section{Estudio de tiempos:}

De acuerdo con Benjamín (1999), el estudio de tiempos es una actividad que implica la técnica de establecer un estándar permisible para realizar una tarea determinada, con base en la medición del contenido del trabajo del método prescrito [14]. Considerando lo anterior, se emplea dicha metodología en el proceso estructurado para fijar estándares que permitan valorar los cambios y mejoras dentro de la ejecución de actividades. Por motivos de salud pública presentados en el año 2020, se decidió aplicar el modelo de análisis de tiempos estructurados (mediante entrevista), lo cual comprende el criterio y experiencia de expertos en el área de investigación buscando valores diferenciales, basados en diversas perspectivas del mismo campo laboral con el fin de asignar una importancia al dato recolectado [14].

Como primera etapa se llevó a cabo la selección de los expertos dispuestos a colaborar con la estimación de tiempos para las actividades previamente documentadas. La información se obtuvo mediante entrevistas virtuales en las cuales se explicó la finalidad de cada actividad y los involucrados aportaron un valor acorde a su experiencia y conocimiento profesional en el área. Además, se recolectó en conjunto con el coordinador logístico los tiempos empleados por la organización en investigación.

Teniendo presente que, en una etapa de valoración de un tiempo se requiere del mayor grado de objetividad, y de acuerdo a las especificaciones de la OIT, se tiene que difícilmente las organizaciones pueden llegar a alcanzar un nivel de producción estándar debido a que cada tarea o actividad ejercida por un colaborador exige esfuerzo y por ende hay que prever ciertos suplementos para resarcir la fatiga con el descanso [15].

Por lo anterior y una vez recolectados la totalidad de los datos, se determinó la tasa de suplementos para cada subproceso con el objetivo de calcular su tiempo estándar. La estimación de estos suplementos se realizó por medio de variables constantes y sugeridas en las fuentes bibliográficas: Las necesidades personales $(4 \%)$, eventualidades $(5 \%)$, políticas de desempeño y especiales $(0 \%)$. Para definir la tasa de fatiga básica se definió en razón de la dificultad o situación en la que se ejecutan las actividades. En seguida se demuestra el impacto causado en cada proceso por los suplementos definidos (Ver Fig. 7).

Finalmente, se procedió a calcular el tiempo estándar para cada actividad, y en general para cada subproceso (Ver figura 8). Como parte del trabajo de análisis, se decidió calcular este tiempo de ejecución para un bien y por actividad. Este factor fue decisivo puesto que el proceso logístico de la empresa de telecomunicaciones tiene una alta tasa de excepciones con respecto a las otras operaciones que se trabajaron con los expertos implicados 


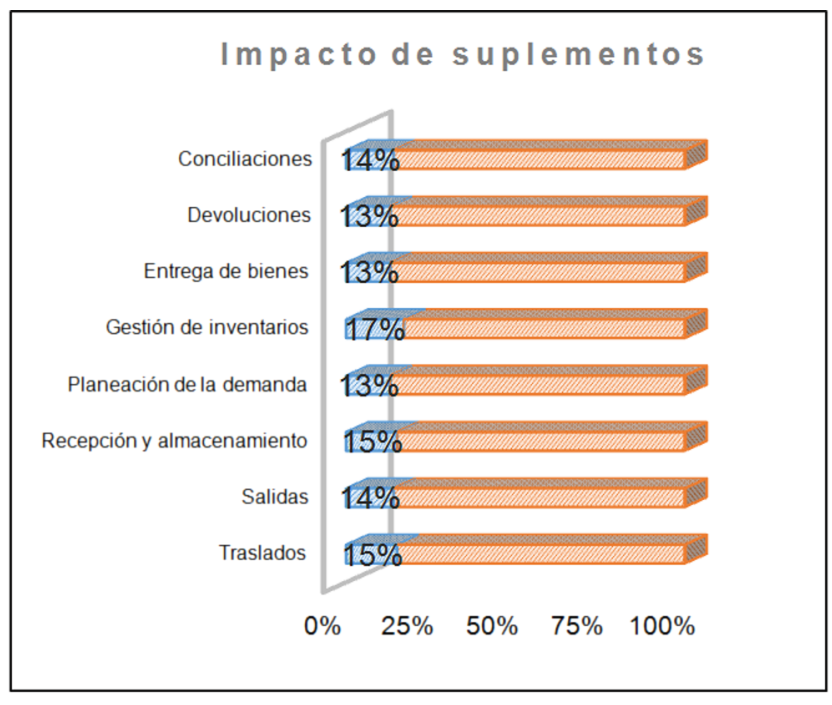

Fig. 7. Porcentaje de suplementos con respecto al tiempo estándar. Fuente: Los autores, 2021

en el estudio, de tal manera se logró relacionar cada una de las actividades sin afectar el tratamiento de datos por la variabilidad de demanda o flujo de bienes.

Se identificó además, las actividades de operación, inspección y auto inspección mediante la elaboración de diagramas de procesos, con el fin de representar los tiempos para cada uno de los casos de forma operacional. Fig. 8

Como resultado, se determinó que dentro de la propuesta de mejora existen oportunidades de optimizar tiempos en el 50\% de los subprocesos logísticos como lo son: Recepción y almacenamiento de bienes, entrega de bienes a los colaboradores, gestión de devoluciones y salidas de bienes. Estos presentaron un déficit en el tiempo de ejecución debido a que poseen actividades que requieren de esfuerzos extensos por la falta de automatización. Los sistemas de información actuales no abarcan en su totalidad las operaciones realizadas y dependen de tareas manuales que generan demoras innecesarias dentro del flujo procedimental. Sin embargo, en los procesos restantes se logró demostrar que a pesar del nivel de análisis, los tiempos estimados están por debajo del estándar, lo que indica la generación de valor en los tiempos y la no necesidad de inversión en mano de obra para ser productivos.

Por último, un $14 \%$ del tiempo debe ser utilizado por los colaboradores para compensar el esfuerzo demandado en cada una de sus actividades dentro del proceso logístico y se recomienda elevar los controles del flujo para garantizar una mayor calidad y confiabilidad en los servicios prestados.

\section{Análisis de impacto}

Con el objetivo de evidenciar el impacto del trabajo realizado en la compañía de telecomunicaciones, se decidió emplear el modelo PEF de tal manera que se pueda traducir los problemas de calidad de un proceso al lenguaje de la empresa, los costos [16]. Esto, para ayudar a la gerencia a evaluar la importancia de los problemas de calidad y las mejoras propuestas que pueden ayudar a reducir o mitigar los valores que implican.

Tiempos organizacionales vs tiempos estándar

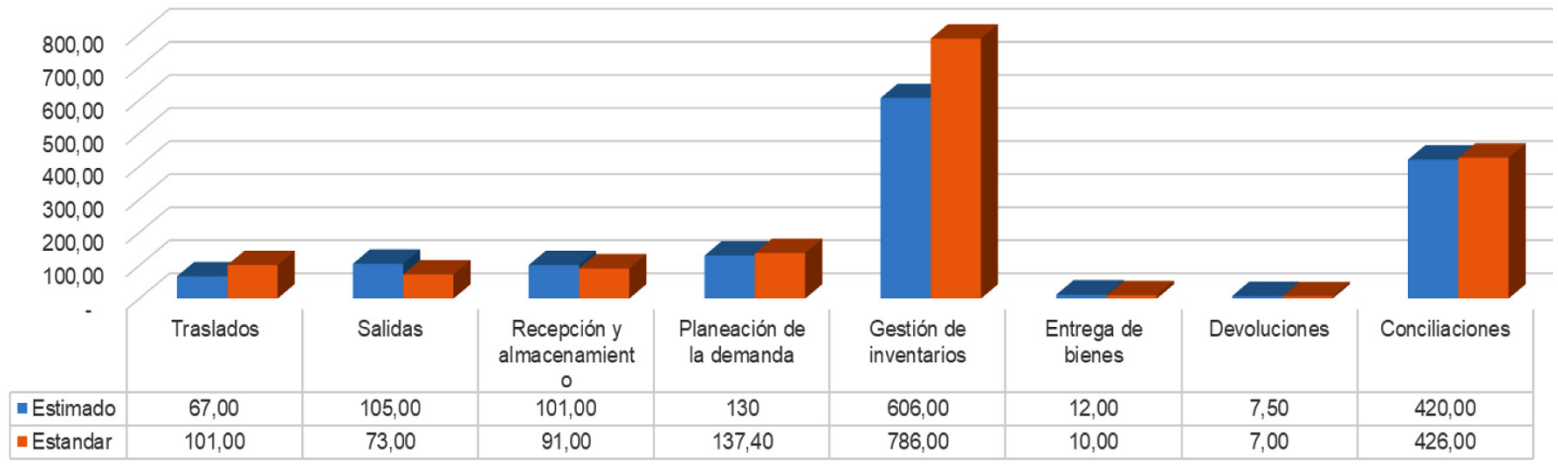

Fig. 8. Comparación entre el tiempo estimado y el tiempo estándar definido en minutos (min]. Fuente: Los autores, 2020 
Debido a que se deseaba impactar al área de Logística y alertar de actividades que deben controlarse de tal manera que se ejecuten bien cada vez que se hagan, el primer paso fue la identificación de los riesgos, teniendo como base aquellos que fueron planteados en la caracterización con el apoyo de los responsables del proceso. Sin embargo, se identificaron riesgos adicionales que están relacionados a cada uno de los subprocesos logísticos de la compañía y que se trataron con los controles definidos en la documentación.

Para identificar los actores se recurrió a metodologías de investigación científica que proponen herramientas cualitativas como lo es la observación y para algunos elementos se aplicó la indagación directa.

De acuerdo a autores como Roberto Hernández [17] la investigación tiene como objetivo no solo comprender procesos y la vinculación de las personas en el desarrollo de las circunstancias, sino identificar problemas y explorar los contextos presentes en las situaciones evaluadas, para lo que se aplicó las estrategias que el sugiere para quién realice observación sin ser experto.

Luego de la identificación de los costos PEF y de los actores involucrados en los procesos logísticos, se logró conseguir el valor por cada uno de los elementos con el fin de priorizar aquellos subprocesos que representan mayor riesgo para la operación y por ende, un mayor costo al incurrir en ellos. De la priorización se obtiene que los subprocesos de planeación de la demanda y solicitud de bienes $(17.5 \%)$, recepción y almacenamiento $(12.5 \%)$, gestión de devoluciones $(12.5 \%)$, gestión de salidas (12.5\%), gestión de traslados $(11.3 \%)$ y conciliación de bienes $(10 \%)$, representan un $76 \%$ de los riesgos del proceso (Ver Fig. 9).

Como resultado de todo el modelo PEF aplicado, y de los actores definidos, se obtuvo finalmente que, la compañía puede presentar grandes costos por hora al incurrir en riesgos como:

- Error u omisión en la identificación de bienes en estado de posible baja, representando costos de más de 5 SMMLV

- Error u omisión de soportes que contemplan conceptos técnicos y/o resultados disciplinarios

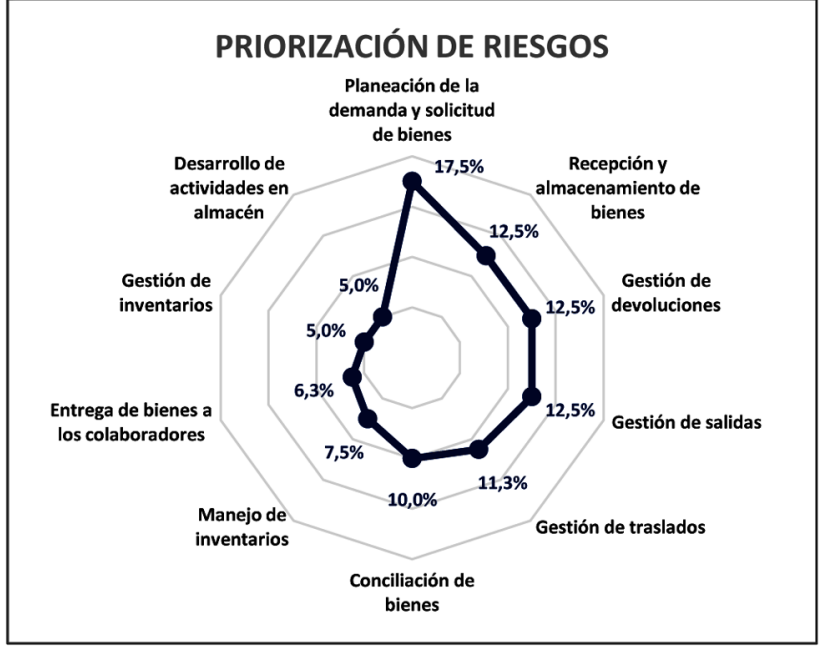

Fig. 9. Priorización de riesgos en los procesos logísticos de la compañía. Fuente: Los autores, 2021.

de bienes, representando costos de más de 5 SMMLV

- Registro incorrecto de los movimientos de inventario en los sistemas de información, representando costos de casi 5 SMMLV

- Pérdida de soportes para la conciliación, representando costos de 4.5 SMMLV

- Errores en la marcación y/o almacenamiento de los bienes, representando costos de más de 4 SMMLV

Como parte del análisis de la distribución de los costos de acuerdo con los elementos PEF propuestos, se tuvo que los costos de no calidad (costos por fallos internos y externos) representan un $61 \%$ del costo total (Ver Fig. 10).

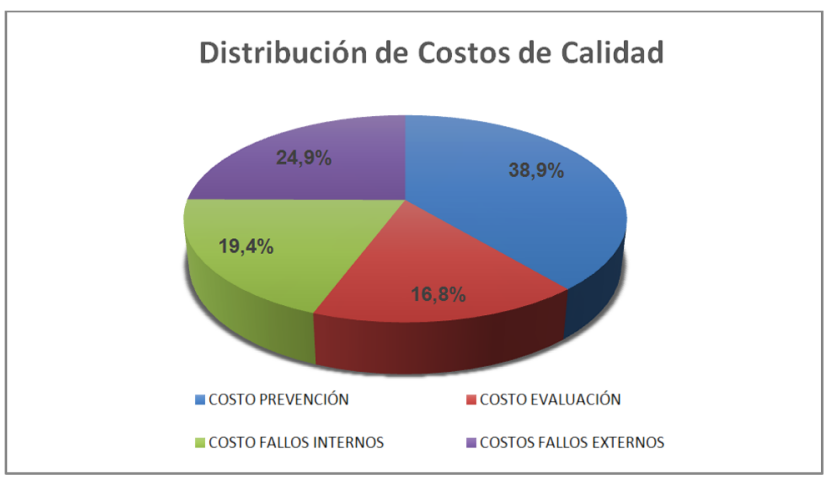

Fig. 10. Distribución del costo total de Calidad. Fuente: Los autores, 2021 
A continuación, (Ver Fig. 11) se observa el comportamiento de los costos de acuerdo a los conceptos PEF. Como tendencia se identifica la importante participación de los costos de prevención en los riesgos detectados, y también se evidencia la importancia de generar estrategias de evaluación para disminuir el riesgo de incurrir en los costos de no calidad, principalmente de fallos externos, puesto que representan en general casi un $25 \%$ de importancia dentro del modelo.

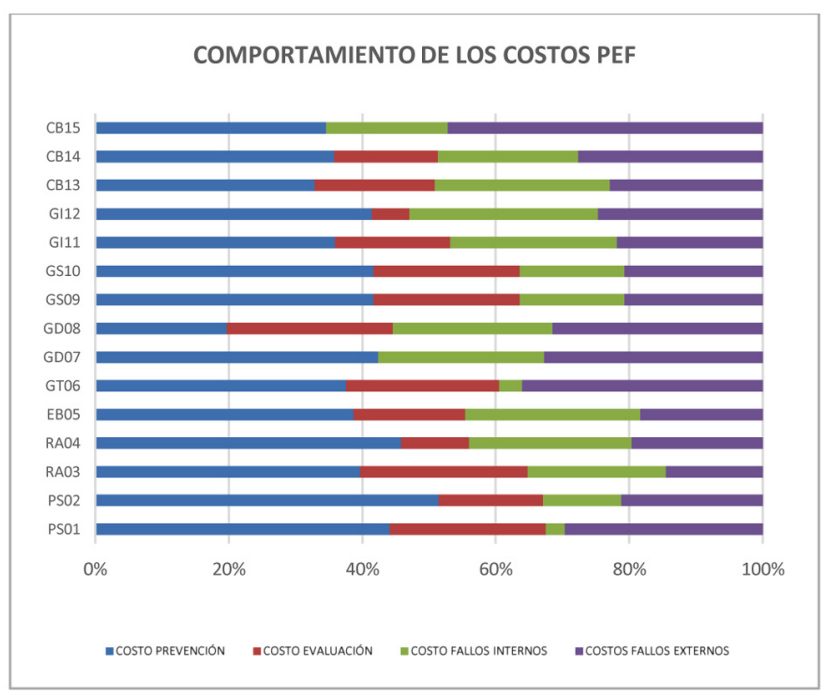

Fig. 11. Comportamiento de los costos totales de calidad. Fuente. Los autores, 2021

\section{Discusión}

Para el desarrollo de la investigación se tomaron como referencia diferentes estados de arte o antecedentes con el fin de contextualizar el trabajo de campo y conocer herramientas aplicadas en trabajos de estandarización, sin embargo, aunque se revisó una cantidad considerable de investigaciones enfocadas en el mismo objetivo se tuvo la dificultad de contar con información o referencias relacionadas con el tema de estandarización en procesos logísticos principalmente en el sector de las telecomunicaciones. Gracias a esta limitante, se optó por adoptar metodologías y herramientas que se aplicaron en otro tipo de procesos logísticos como los correspondientes a distribución o empaque de empresas productoras.

Para un entendimiento más detallado, una de las referencias en las que se basó este trabajo fue una investigación realizada por Mira de Jesús [18] donde se aplicó una herramienta de evaluación factorial con el fin de detectar las necesidades de estandarización de los procesos de operación de una compañía retail. De acuerdo con los resultados, para los procesos que se identificaron, se procedió a registrar los subprocesos y actividades asociadas y luego a los responsables a través de herramientas de observación y medición, para finalmente proceder a la elaboración de la caracterización.

A pesar de que esta metodología demuestra un análisis inicial para identificar los procesos de operación que debían ser estandarizados y una serie de pasos que estructuran la normalización, no se tuvo en cuenta ningún tipo de registro o base para realizar este proceso y tampoco se evidencia la importancia de impactar todo el trabajo operacional de la compañía implicada.

De acuerdo con las necesidades que se evidenciaron en la compañía del trabajo que se expone en este artículo, al contrario, se tuvo primero como base la información que ya se encontraba documentada y con ayuda de esto y también de herramientas de medición y observación se hizo el debido levantamiento de información de tal forma que se lograra identificar las posibles mejoras para finalmente plasmarlas en la caracterización del proceso de logística.

Para la implementación de indicadores, se evidenció que en un trabajo de investigación llevado a cabo por Ramírez Carolina [19] se diseñó un sistema de gestión de indicadores según la metodología del Cuadro de Mando Integral para medir los procesos operativos del área de logística de distribución aplicando un modelo de brainstorming para definir los procesos que debían ser evaluados. Para el trabajo en la compañía de telecomunicaciones los autores se basaron en las necesidades expuestas por los dueños del proceso de logística, mejora continua y la dirección para definir dichos indicadores y enfocarlos principalmente en la política de calidad.

En cuanto a la toma de tiempos para el estudio del trabajo, este proyecto se enfrentó a una situación de contingencia por temas de salubridad que provocó cambios a nivel mundial en el modo de trabajo, por esta razón, se decidió emplear el modelo de toma de tiempos estructurados para la

Rev. Ingeniería, Matemáticas y Ciencias de la Información Vol. 8 / Núm. 15 / enero - junio de 2021; pág. 33-44 
ejecución de esta fase. Contar con expertos que conozcan los procesos relacionados a la administración de bienes de servicios de telecomunicaciones no fue una tarea fácil, por lo que se explicó a través de un proceso de entrevista y de manera macro el sentido de cada una de las actividades y el objetivo de cada subproceso para lograr la vinculación de los expertos que ayudaron significativamente en la continuidad de esta investigación.

Finalmente, aplicar el modelo PEF, para evaluar el impacto de costos de no calidad, ha sido una decisión de los autores para generar valor al proyecto y aportar información importante a la compañía, pues, en otros trabajos tomados como referencia no se evidenció un análisis parecido. Para llevarlo a cabo, se hizo investigación de la aplicación de esta evaluación en otros sectores como el que realizó Cardona (2013) y sus fundamentos [20] [21], lo que facilitó la adopción a las necesidades del proceso logístico en cuestión.

En definitiva, vale la pena resaltar, que este proyecto sirve como fundamento teórico para futuras investigaciones que aborden el proceso de la estandarización de procesos logísticos en el sector de las telecomunicaciones. También, sirve como un aporte documental que se brinda al sector, y que puede ser de gran beneficio para adoptar nuevas metodologías de trabajo.

\section{Conclusiones}

La propuesta de estandarización permitió aplicar herramientas de ingeniería en el área de logística que apoyaron la metodología y ofrecieron resultados que facilitan la toma de decisiones para optimizar los procesos y recursos.

Se suplió la necesidad de un sistema de gestión documental actualizado y la estructuración de indicadores de gestión que ofreciera seguridad a los dueños del proceso y la dirección.

En lo referente al estudio de tiempos se permitió considerar la opinión de varios expertos en los temas propuestos, además de establecer un patrón de comparación con el fin de que la institución evalué sus procesos frente a otras instituciones y así mismo identificar estrategias de mejora en los puntos críticos detectados.
En cuanto a la metodología PEF se mostró la necesidad de evaluar las actividades relacionadas a los riesgos con el fin de identificar el impacto de los costos de calidad o no calidad y se amplió el panorama para la compañía dejándole evidencia de la importancia de invertir en actividades de prevención, lo que disminuiría considerablemente los costos totales ya que son los que más participan dentro del modelo y se alejaría de la posibilidad de que se presenten fallas en los servicios.

Se propició un modelo de estandarización documental alineado a los objetivos institucionales que permitió la articulación, el cambio y la medición de los procesos de la compañía enfocados en la gestión por procesos y mejora continua.

\section{REFERENCIAS}

[1] J. Zarategui, La gestión por procesos, su papel e importancia en la empresa. Pg. 81-88. 1999.

[2] Ministerio de Tecnologías de la Información y las Comunicaciones. Estudio del sector. Recuperado del Sitio Web de la MINTIC del Gobierno de Colombia: https:/ / colombiatic.mintic.gov.co/679/ w3-channel.html. 2019.

[3] Departamento Administrativo Nacional de Estadística (DANE). Indicadores básicos de TIC en hogares y empresas. Recuperado del Sitio Web de la MINTIC del Gobierno de Colombia: https:// colombiatic.mintic.gov.co/679/w3-channel.htm. 2019.

[4] TIC Panorama, Comportamiento macroeconómico del Sector TIC en Colombia. Recuperado del sitio web de la MINTIC del Gobierno de Colombia: https:/ / colombiatic.mintic.gov.co. 2015.

[5] Universidad Nacional, "Guía metodológica para el estudio de cargas de trabajo 2013". Pg. 1-10. 2013.

[6] S. E. Miguel, M. E. Palmer, J. M. Albarracín, C. Romano, Una revisión de las Tablas de Suplementos de la Organización Internacional del Trabajo. 2017.

[7] S. Gracia, L. A. Dzul, Modelo PEF de costes de la calidad como herramienta de gestión en empresas constructoras: Una visión actual. Recuperado de h t t p s : / / s c i elo.conicy t.c l / scielo.php?script $=$ sci_arttext\&pid $=$ S071850732007000100005. 2007.

[8] K. Ramírez, V. Álvaro, Prácticas de mejora continua, con enfoque Kaizen, en empresas del distrito metropolitano de Quito: Un estudio exploratorio. 
Intangible Capital, 13(2), 479-497. https:/ / doi.org/ 10.3926/ic.901. 2017.

[9] K. Tapia, J. Rodolfo, Un sistema de costos de calidad piloto aplicando el modelo PEF en la empresa Flex N Gate Hermosillo (Master's thesis, Keith Tapia, Jesús Rodolfo). 2012.

[10] Organización Internacional del Trabajo (OIT). Recuperado del sitio web de la Cancillería de Colombia, https://www.cancilleria.gov.co/en/node/ 406.

[11] R. Bermúdez, A. Millan, Metodología para el Mejoramiento en los Procesos de Dirección de Proyectos del Fondo de Prevención y Atención de Emergencias - FOPAE. 2013.

[12] H. Gutiérrez, Calidad Total y Productividad. México. McGraw-Hill/Interamericana Editores, S.A. de C.V. 2010.

[13] J. Pérez, Los indicadores de gestión. Recuperado del sitio web https:

//www.visionadministrativa.info

[14] W. Benjamín, Niebel. Ingeniería industrial - Métodos, tiempos y movimientos. Alfaomega. (9a edición). 1999.

[15] Organización Internacional del Trabajo (OIT), Introducción al estudio del trabajo. (4a. Edición). Limusa, Grupo Noriega Editores. 2004.
[16] A. R. Aguirre, C. Marín. Análisis de los costos de calidad y no calidad de la secretaria de planeación municipal (Alcaldía de Pereira sector central). especialización en gestión de la calidad y normalización técnica. Disponible en: URL:http:/ / repositorio.utp.edu.co/dspace/handle/11059/ 1531. 2008.

[17] R. Hernández, Metodología de la investigación. México, Mc Graw-Hill Editores, pp. 453-455. 1998.

[18] C. Mira de Jesús, Estandarización de procesos como herramienta de mejora a la calidad de procesos administrativos. Universidad Nacional Autónoma de México. Facultad de ingeniería. 2016.

[19] C. Ramírez, Diseño e implementación de indicadores de gestión logísticos en el área logística de distribución en la empresa Carvajal Pulpa y Papel S.A. Universidad Autónoma de Occidente. Facultad de ingeniería. 2012.

[20] S. Herring, B. Jones, B. Bailey, Idea generation techniques among creative professionals. Proceedings of the 42nd Hawaii International conference on system sciences. 2009.

[21] L. Cardona, Importancia de los costos de la calidad y no calidad en las empresas de salud como herramienta de gestión para la competitividad. Disponible en: https://doi.org/10.21158/ 01208160.n67.2010.486. 2013. 